\title{
Comportamiento a la corrosión del acero 316L sinterizado con distinto grado de porosidad
}

\author{
L. Soria ${ }^{(*)}$, F. Gómez ${ }^{(*)}$, J.M. Gallardo ${ }^{(*)}$ y E.J. Herrera ${ }^{(*)}$ \\ Resumen A partir de polvos de acero inoxidable AISI 316L, se han preparado muestras con distinto grado de \\ porosidad, entre el 9 y el $40 \%$, asegurando que la superficie externa no resulta alterada durante el pro- \\ cesado pulvimetalúrgico. Junto a muestras de chapa laminada de la misma composición, han sido \\ sometidas a ensayos de inmersión y a ensayos electroquímicos de corrosión. Estudios cuantitativos de \\ la evolución de la porosidad superficial, antes y después de los ensayos, permiten plantear diversas \\ hipótesis acerca de los mecanismos de corrosión actuantes en función de la porosidad de las piezas. \\ Palabras clave: Pulvimetalurgia. Acero inoxidable. Ensayos de corrosión. Porosidad.

\section{Corrosion behaviour of a sintered 316L steel with different porosity grades}

\begin{abstract}
AISI 316L sintered samples, with porosities ranging from 9 to $40 \%$, and without alloying losses at the surface, have been prepared. Those samples, along with conventional (rolled) steel samples, have been subjected to electrochemical and inmersion corrosion tests. According to porosity size quantitative measurements, before and after corrosion tests, two corrosion mechanisms, general and pitting, are proposed depending on the initial porosity.
\end{abstract}

Keywords: Powder metallurgy. Stainless steel. Corrosion tests. Porosity.

\section{INTRODUCCIÓN}

Las aplicaciones de los aceros inoxidables pulvimetalúrgicos (PM) están limitadas por las dificultades en obtener las mismas propiedades que en los aceros clásicos. La resistencia a la corrosión aparece como el principal problema que limita el uso de este tipo de materiales.

El efecto del tamaño y porcentaje de porosidad sobre la resistencia a la corrosión es conocido (1-5), aunque existen discrepancias acerca de su signo, según el medio corrosivo y el tipo de ensayo, químico o electroquímico, utilizado (6). Para una misma densidad, otros factores como el contenido de carbono, nitrógeno u oxígeno (7), el carácter conectado o interconectado de la porosidad (8), el tamaño de los poros individuales (9), la uniformidad de tamaño de poro según el proceso de fabricación del polvo (10) etc., modifican el efecto obser-

(*) Grupo de Metalurgia e Ingeniería de los Materiales. E.S. de Ingenieros, Avda. de los Descubrimientos s/n. 41092-Sevilla (España). vado de la porosidad en la resistencia a la corrosión. También la composición química de la superficie, tanto de los polvos como del producto final, influye sobre la resistencia a la corrosión. La presencia de capas de óxidos superficiales $\left(\mathrm{SiO}_{2}\right.$, $\mathrm{C}_{2} \mathrm{O}_{3}, \mathrm{Fe}_{2} \mathrm{O}_{3}$, etc.) en los polvos dificulta la sinterización (11) y favorece la corrosión por picaduras al resultar agrietada durante la compactación (9). La eliminación de dichas capas se facilita mediante el empleo de elevadas presiones de compactación (1 y 12) y elevadas temperaturas de sinterización (11) en atmósferas con bajo punto de rocío (13). En este sentido, atmósferas de hidrógeno con puntos de rocío inferiores a $-34{ }^{\circ} \mathrm{C}$, amoníaco disociado con puntos de rocío inferiores a $-62{ }^{\circ} \mathrm{C}$ o vacío a presiones inferiores a $10^{-2}-10^{-3}$ mbar se consideran satisfactorias.

Además, una defectuosa eliminación del lubricante, bajas velocidades de enfriamiento tras el procesado -sobre todo unido a contenidos elevados de carbono o nitrógeno, en el metal o la atmósfera de sinterización (1 y 14)-, etc., son factores que favorecen fenómenos corrosivos posteriores o durante el propio procesado (6). 


\section{PROCEDIMIENTO EXPERIMENTAL}

Se han preparado muestras cilíndricas de $12 \mathrm{~mm}$ de diámetro y $5 \mathrm{~mm}$ de espesor, mediante compactación en frío con lubricación en pared. Se han empleado presiones de 100, 400, 700 y $1.000 \mathrm{MPa}$, al objeto de obtener diferentes porosidades finales. Se ha realizado una desgasificación a $425{ }^{\circ} \mathrm{C}$, al aire, durante $1 \mathrm{~h}$. La sinterización, en atmósfera dinámica de argón a 0,8 mbar, se ha llevado a cabo a $1.250{ }^{\circ} \mathrm{C}$ durante $1 \mathrm{~h}$. Las muestras se han enfriado, desde la temperatura de sinterización, en corriente de argón. Mediante el procedimiento descrito, las muestras experimentan una densificación aceptable, sin que la superficie muestre pérdidas detectables de elementos de aleación (15).

Se han determinado la densidad y la porosidad medias, así como algunas características geométricas de la porosidad superficial.

En micrografías de la superficie de las muestras, realizadas por microscopía electrónica de barrido con detector BSE, los poros superficiales más profundos aparecen negros, ya que no se recibe señal de electrones del fondo de los mismos. De esta forma, se han segmentado y determinado cuantitativamente el porcentaje de porosidad superficial y la distribución de tamaños (áreas) de dichos poros.

Por su parte, en micrografías ópticas de secciones transversales a la superficie a ensayar, se han cuantificado la anchura y profundidad máximas de los poros superficiales. Para ello, en la micrografía, se ha definido como contorno del poro superficial aquella área vacía, rodeada de metal, y abierta a la superficie externa.

Antes de someter las probetas a ensayo, han sido tratadas superficialmente con un desoxidante para eliminar, en su caso, ligeras irisaciones que puedan quedar tras la sinterización.

Las muestras han sido sometidas a ensayos de corrosión electroquímicos y por inmersión. Los ensayos electroquímicos se han realizado según la norma ASTM G61, en $\mathrm{NaCl}$ al 3,56 \% desaireado, a $25^{\circ} \mathrm{C}$. Las probetas han sido embutidas, pero sólo hasta la mitad de su espesor, modificando así ligeramente una técnica de ensayo que evita o disminuye la aparición de corrosión en hendiduras (16).

Los ensayos de inmersión durante 72 h en $6 \%$ $\mathrm{FeCl}_{3}$ se han realizado según la norma ASTM G48. Como quiera que dicho ensayo es muy agresivo para los aceros sinterizados, se han repetido las experiencias con tiempos de inmersión de 5 h (17).

\section{RESULTADOS Y DISCUSIÓN}

Las densidades finales medias para las muestras comprimidas a 100, 400, 700 y $1.000 \mathrm{MPa}$ son, respectivamente de $4,77,6,25,6,90$ y $7,27 \mathrm{~g} / \mathrm{cm}^{3}$, lo que corresponde a porosidades del $40,4,21,9,13,7$ y $9,2 \%$.

Los ensayos electroquímicos muestran que, incluso en las probetas más densas, no se obtiene pasivación de la mismas, aun llegando a densidades de corriente de $100 \mathrm{~mA} / \mathrm{cm}^{2}$ (Fig.1), en contraste con lo observado en el acero convencional.

Por su parte, los ensayos de inmersión muestran un peor comportamiento de los aceros pulvimetalúrgicos, con velocidades de corrosión hasta 200 veces superiores que el acero convencional. Puede señalarse (Fig.2) que la velocidad de corrosión de los aceros pulvimetalúrgicos disminuye con el tiempo, es decir, se mide una menor velocidad en el ensayo ASTM G48 normalizado que en el modificado a $5 \mathrm{~h}$. Lo contrario puede decirse de los aceros convencionales. Además, el ensayo modificado discrimina mejor entre las probetas sinterizadas con diferente porosidad final.

El cambio en la porosidad superficial, antes y después del ensayo ASTM G48 modificado, medido en micrografías SEM, puede deducirse por comparación 'de las figuras $3 \mathrm{a}$ y $3 \mathrm{~b}$. En las probetas menos densas no se producen cambios significativos ni en el porcentaje ni en la distribución por tamaños de la porosidad superficial. En las probetas más densas, en cambio, se aprecia un aumento tanto del porcentaje de porosidad, como del área media de los poros. Así, la porosidad superficial, antes y después del ensayo es de 8,1 y $7,9 \%(100 \mathrm{MPa}) ; 5,2$ y $5,1 \%$ (400 MPa); 3,1 y $3,4 \%$ (700 MPa) y 2,3 y $4,1 \%$ (1.000 MPa) para las muestras comprimidas a las presiones señaladas. Por su parte, el cincuentil de las áreas de poro medidas en superficie pasa, antes y después del ensayo, de 0,9 a $0,8 \mu \mathrm{m}^{2}(100 \mathrm{MPa})$; de

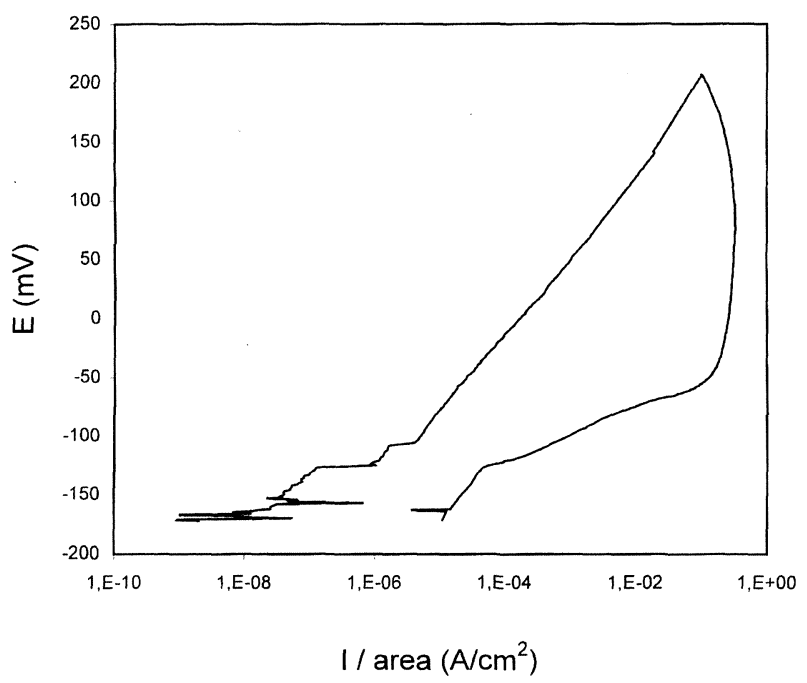

FIG. 1. - Curva de polarización del acero 316L pulvimetalúrgico preparado con $1.000 \mathrm{MPa}$.

FIG. 1. - Sintered 316 L steel polarization behaviour. Samples were prepared with 1,000 MPa. 


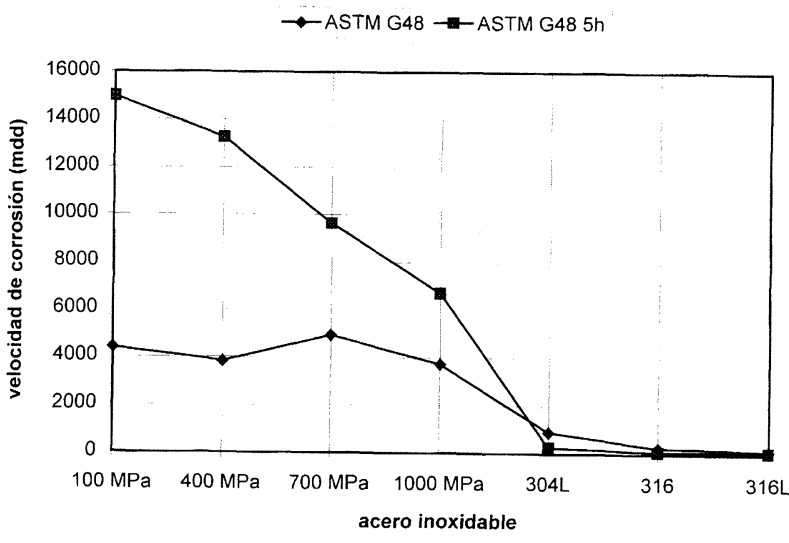

FIG. 2.- Velocidades de corrosión de los aceros inoxidables convencional y pulvimetalúrgico en el ensayo ASTM G48 normal y modificado.

FIG. 2.- Corrosion rates for rolled and PM steels after standard and modified ASTM G48 tests.

0,5 a $0,3 \mu \mathrm{m}^{2}$ (400 MPa); de 0,3 a $0,4 \mu \mathrm{m}^{2}(700$ $\mathrm{MPa})$ y de 0,2 a $0,4 \mu \mathrm{m}^{2}(1.000 \mathrm{MPa})$.

Un comportamiento equivalente se observa al comparar las medidas de porosidad superficial realizadas mediante microscopía óptica, antes y después del ensayo ASTM G48 modificado. Las figuras $4 \mathrm{a}$ y $4 \mathrm{~b}$ presentan, respectivamente, la distribución de la anchura y la profundidad de los poros superficiales de muestras comprimidas a 100 $\mathrm{MPa}$, tanto antes como después del ensayo ASTM
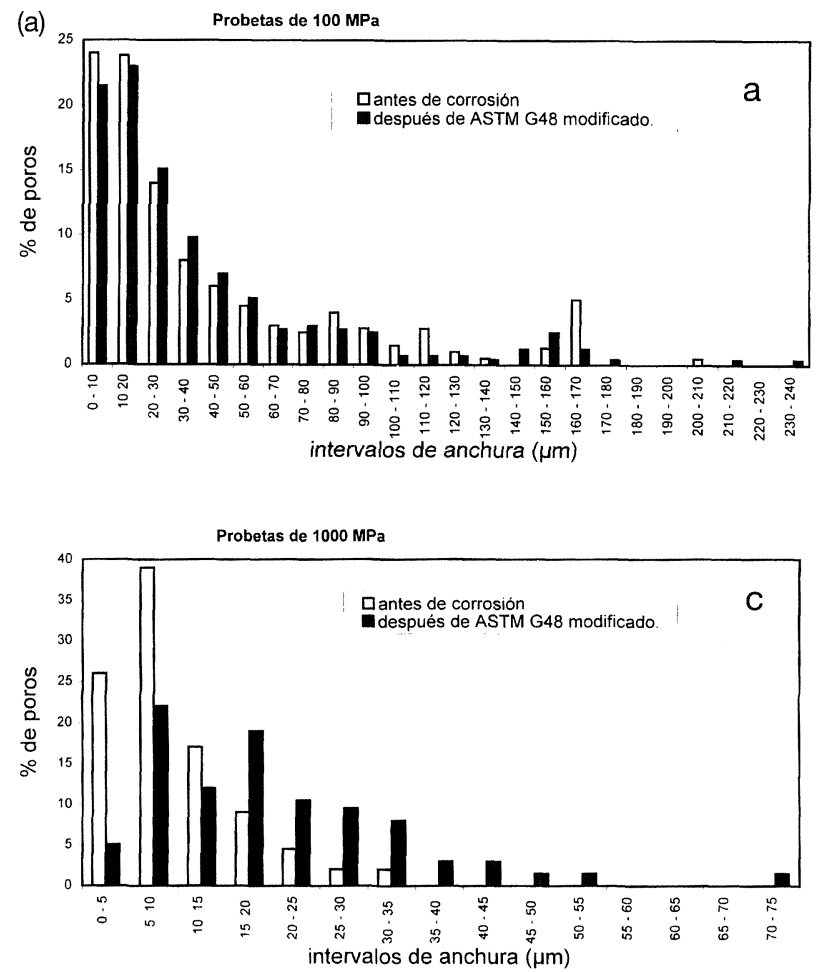
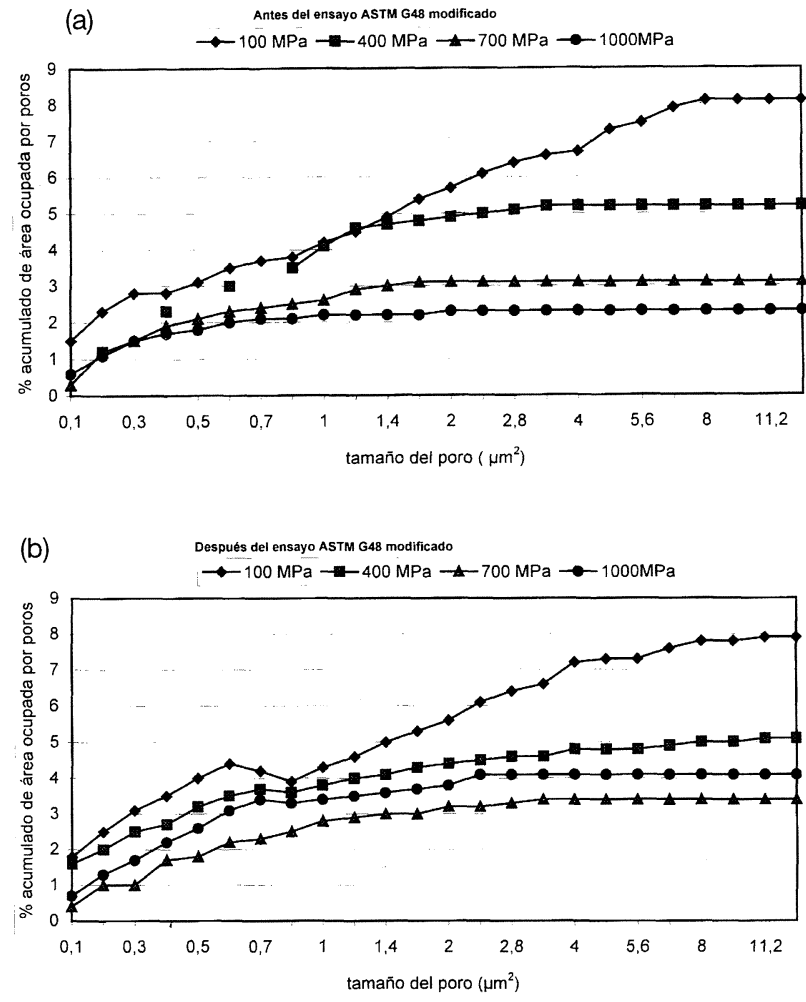

FIG. 3.- Distribución de tamaños de la porosidad superficial $\left(\mu \mathrm{m}^{2}\right)$ medidos en micrografía SEM.

Fig. 3.- Size distribution $\left(\mu m^{2}\right)$ of surface porosity as measured in SEM micrographs.
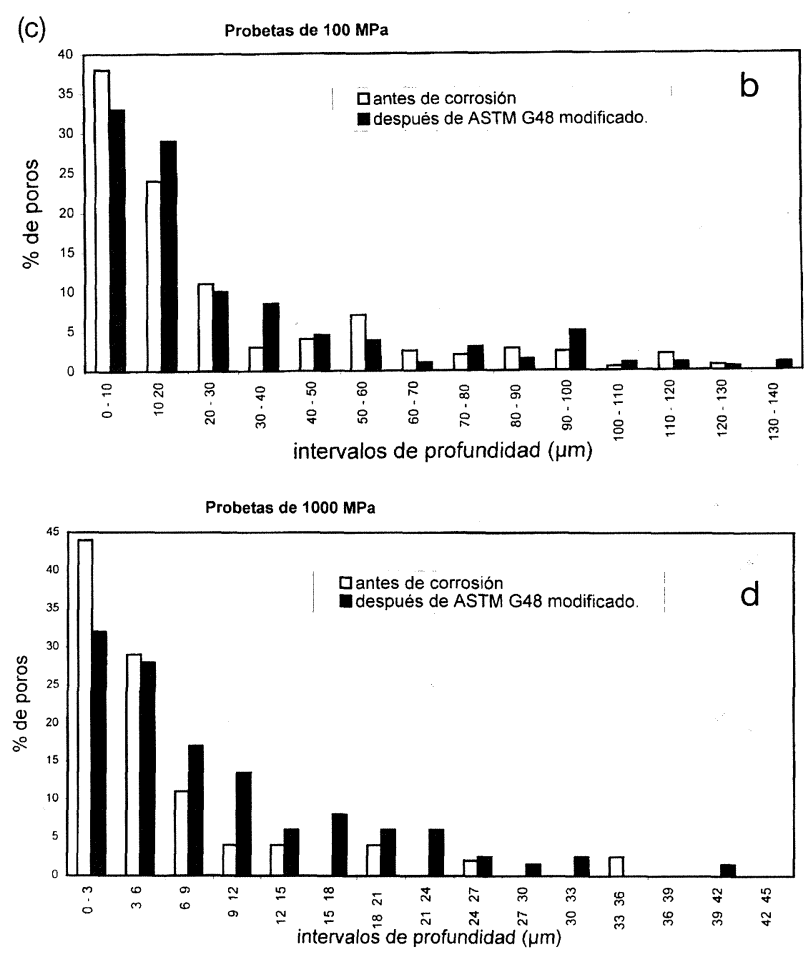

FIG. 4.- Distribución de las dimensiones de los poros superficiales medidas mediante microscopía óptica.

FIG. 4.- Size distribution of surface porosity as measured in optical micrographs. 
G48 modificado. No puede deducirse una evolución clara de las dimensiones de los poros durante el ensayo de corrosión. En las figuras $4 \mathrm{c}$ y $4 \mathrm{~d}$ se representan las mismas variables para las muestras comprimidas a $1.000 \mathrm{MPa}$. Aquí, se aprecia que la corrosión ha producido un claro aumento de la anchura y de la profundidad de los poros. Comportamientos intermedios se han observado en las muestras comprimidas a presiones intermedias entre las anteriores.

De acuerdo con las observaciones anteriores, se estima que las elevadas tasas de velocidad de corrosión observadas en las muestras menos densas se producen por corrosión uniforme de las capas superficiales de las mismas, que presentan una elevada porosidad, suficientemente abierta, como para asegurar una buena renovación de las especies implicadas en la corrosión. Este mecanismo va modificándose conforme la porosidad superficial es más escasa o más cerrada; es decir, para las probetas más densas. En este último caso, los poros superficiales comienzan a actuar como verdaderos núcleos de picaduras, favoreciendo los conocidos fenómenos corrosivos en el interior de las mismas. Por ello, aunque las velocidades netas de corrosión son menores en los aceros más densos, el aumento observado en el tamaño y profundidad de los poros es sensiblemente mayor.

En este sentido, debe entenderse que las probetas compactadas a $700 \mathrm{MPa}$ presenten mayor pérdida de peso en el ensayo de inmersión que las preparadas a $1.000 \mathrm{MPa} y$, sin embargo, estas últimas desarrollen una mayor "porosidad" (= picaduras) superficial que las primeras. Es decir, de acuerdo con las experiencias realizadas, el cambio del mecanismo de corrosión uniforme a corrosión por picaduras se produce cuando la porosidad disminuye por debajo de un 15 $\%$, aproximadamente (porosidad superficial medida por SEM inferior al $2.5 \%$ ).

En todo caso, dado que las picaduras parecen generarse en la porosidad previa, no tendría sentido hablar de nucleación de picaduras o, lo que es lo mismo, de potencial de picaduras. Este fenómeno queda corroborado por los resultados electroquímicos realizados. Otros autores (13) sí han mostrado la posibilidad de pasivar el acero inoxidable pulvimetalúrgico, pero cuando la porosidad no es interconectada, es decir, para densidades superiores al $93 \%$, aproximadamente.

\section{CONCLUSIONES}

Se han preparado muestras pulvimetalúrgicas exentas de oxidación superficial con porosidades entre el 9 y el $40 \%$. Junto a muestras de acero convencional han sido sometidas a ensayos de corrosión electroquímicos y de inmersión. No se aprecia pasi- vación en los ensayos electroquímicos de los aceros pulvimetalúrgicos y su velocidad de corrosión es considerablemente más alta que la de los convencionales, disminuyendo con el tiempo de ensayo.

El estudio de la evolución del tamaño de los poros superficiales permite deducir que las muestras con más del $15 \%$ de porosidad sufren una corrosión de tipo uniforme, mientras que, por debajo de dicho porcentaje de porosidad, comienza a aparecer corrosión por picadura.

\section{Agradecimiento}

Los autores expresan su agradecimiento a Mlle. Sandrine Falipou por la realización de los ensayos de corrosión. Este trabajo cuenta con la financiación del proyecto MAT 96 - 2311.

\section{REFERENCIAS}

(1) Fories Jones, R.M. Prog. Powder Metall. 30, 1974: 2550.

(2) Raghu, T., Malhotra, S.N., Ramakrishnan, P. Br. Corr. J., 23, 1988: 109.

(3) Raghu, T., Malhotra, S.N., Ramakrishnan, P. Corrosion 45, 1989: 109.

(4) Fedrizzi, L., Molari, A., Delforian, F., Ciaghi, L. y BONORA, P.L. Corrosion 46, 1990: 672.

(5) Rosenfeld, I.L., Staehle, R.W., Brown, B.F., Kruger, J., Agrawal, A. Localized corrosion, Ed. NACE-3, Houston (Texas, EE.UU.) 1974.

(6) Metals Handbook, Corrosion, 9th edition, 13.

(7) Ranninger, C., Torres, A., Aparicio, M.L., Ruiz, J.M., Torralba, J.M. y Martinez, R. Key Engineering Materials, Trans. Techn. Publ. Ed. Madrás (India), 36, 1989: 241-249.

(8) Palmateer, R.F. y Kulkarni, K.M. Proc. 1991 AMPI/MPIF Powder Metallurgy Conf., Chicago (ILL., EE.UU.), 9-12 Jun. 1991: 79-90.

(9) Contreras, W., Demestre, M., Torralba, J.M., Ruiz, J.M., Torres, A. y Ranninger, C. Rev. Iberoam. Corros. Prot., 18 (2-6), 1987: 145-148.

(10) Malhotra, S.N., Mohan, A. y Ramakrishnam, P. Trans. PMAI, 17, dic. 1990: 33-42.

(11) Ro, D.H. y KlaR, E. Modern Develop. Powder Metall., 13, 1980: 247-285

(12) Stainless Steel Powders. Seminario preparado por Hoeganaes Sponge Iron Corporation, Hoganas Billesholms AB, Detroit (Mich. EE.UU.), Feb. 1965.

(13) Tunberg, T., Nyborg, L. y Liu, C.X. Industrial Heating, 59 (11), 1992: 37-42.

(14) Nayar, H.S., German, R.M. y Johnson, W.R. Modern Develop. Powder Metall. 15, 1981: 255-265.

(15) Gomez, F., Gallardo, J.M. y Herrera, E.J. $8^{\circ}$ Cong. Nac. de Ciencia y Tecnología Metalúrgicas, Madrid, Mayo 1998.

(16) Soria, L. y Herrera, E.J. Rev. Metal. Madrid, 27 (6), 1991: 371-379.

(17) Soria, L. y Herrera, E.J., Rev. Iberoam. Corros. Prot., 21 (5), 1990: 177-182. 